\title{
Clinical features of pregnancy and delivery after IVF
}

\begin{abstract}
The number of multiple pregnancies achieved by In vitro fertilization is steadily decreasing over the last years. This leads to increased interest in the course and complications of singleton IVF pregnancies. The purpose of this review is to examine the specific features of the second half of pregnancy, delivery and the newborn in singleton pregnancies achieved by In vitro fertilization. Key findings include pregnancy duration, frequency of preterm birth, low birth weight and small for gestational age neonates. Complications of pregnancy - preeclampsia, gestational diabetes and placenta previa - were also assessed. Research is done according to the method of delivery and the condition of the newborn from these pregnancies.
\end{abstract}

Keywords: IVF, preterm birth, lbw, sga, placenta previa, cesarean section
Volume 10 Issue 4 - 202 I

\author{
Ingilizova G,' Kovachev E, ${ }^{2}$ Ninova $\mathrm{M}^{3}$ \\ " "Vita" Multidisciplinary Hospital for Active Treatment, Sofia, \\ Bulgaria \\ ${ }^{2}$ Department of Obstetrics and Gynecology, Medical University \\ of Varna, Bulgaria \\ ${ }^{3}$ Alexandrovska University Hospital, Sofia, Bulgaria
}

Correspondence: Ingilizova G, “Vita” Multidisciplinary Hospital for Active Treatment, Sofia, Bulgaria, Tel +359887204480,

Emailingilizova@mail.bg

Received: May 18,2021 | Published: August 31,2021
Abbreviations: ART, assisted reproductive technologies; NICU, neonatal intensive care unit; COHS, controlled ovarian hyperstimulation; ET, embryo transfer; Fresh ET, ET during COHS; FET, embryo transfer of frozen / thawed embryos; ICSI, intra cytoplasmic sperm injection; IVF, In vitro fertilization; IUI, intrauterine insemination; LBW, low birth weight; PTB, preterm birth; SGA, small for gestational age; PE, preeclampsia; GDM, gestational diabetes mellitus

\section{Introduction}

For a long time, the development of IVF has been the subject of science fiction and animal experiments. In 1961, the world's first laparoscopic puncture of follicles to obtain eggs was described. The first pregnancy after IVF was reported by the team of Prof. Carl Wood and John Leeton in Melbourne, Australia. Unfortunately, pregnancy ends in early miscarriage. Finally, it came to July 25, 1978, when the world's first child after IVF was born - Louise Brown. Since then, millions of children have been conceived by this method or its modifications. Today newborns from pregnancies after IVF have reached $5 \%$ in some countries. ${ }^{1}$ The purpose of this review was to analyze the course of pregnancy and delivery in singleton IVF pregnancies and to compare it with the course of spontaneous ones. We also wanted to study the available data on the influence of specific elements of the IVF procedure on the outcome of pregnancy.

\section{Course of pregnancy achieved by IVF}

The number of women giving birth after different methods of assisted reproduction is steadily increasing worldwide. Numerous studies have found poorer obstetric outcome in pregnancies achieved by IVF compered to spontaneous ones. Various factors such as concomitant pathology in women, the method of pregnancy, the procedure itself, advanced maternal age as well as the causes of infertility are studied. Many studies have concluded that, compared to spontaneous singleton pregnancies, those achieved through assisted reproductive technologies are more risky in terms of some complications for the mother and fetus, as well as for a less favorable perinatal outcome. ${ }^{1}$ Women who needs IVF usually are older than fertile women. ${ }^{2}$ Maternal age over 35 years is associated with reduced fertility, increased risk of chromosomal abnormalities, miscarriages, multiple pregnancies, low birth weight, gestational diabetes and preeclampsia during pregnancy.
Preterm birth is a serious and current problem in clinical practice. IVF with its various modifications is also considered a risk factor for preterm birth and low birth weight. According to some authors, ${ }^{2,4}$ IVF ranks fifth as a risk factor for preterm birth in singleton IVF pregnancies, after factors such as chronic kidney disease, cervical conization, previous preterm birth, and uterine abnormalities. IVF pregnancies carry a 1.5 times higher risk of preterm birth than spontaneous births singleton pregnancies. ${ }^{4}$ After birth of the first child, IVF has become the standard method of treatment for various factors leading to infertility. Later reproduction and easier access to treatment led to a significant increase in the use of IVF. Initially, studies has been focused mainly on the efficacy of the method and multiple pregnancies. Later, the focus shifted to the effect of IVF on the patient's health, pregnancy and the child's condition. Studies comparing the perinatal outcome of pregnancies after IVF with spontaneous pregnancies have been performed. Most studies found an increased incidence of preterm birth, defined as a birth before the 37th week of pregnancy, low birth weight, defined as a birth weight $<2500$ $\mathrm{g}$ and a small gestational age newborn, defined as the birth of a fetus weighted less than 10th percentile for the respective gestational age. ${ }^{5,6}$ A small number of studies did not find such a link. These problems are essential for clinical practice as they determine the incidence of neonatal morbidity and mortality. ${ }^{7}$

The first major systematic studies examining the impact of IVF on pregnancy and childbirth concluded, that singleton IVF pregnancies compared with spontaneous singleton pregnancies more often lead to preterm birth, early preterm birth $(<32$ w.g.), low birth weight, very low birth weight $(<1500$ g.) and SGA. Early preterm birth, spontaneous preterm birth and NICU admission are also more common in pregnancies after IVF. ${ }^{8}$ In general, the risks for multiple pregnancies after IVF remain much higher, but attention should also be paid to the possibility of a worse outcome in singleton pregnancies after IVF.

A large meta-analysis compared singleton IVF pregnancies with spontaneous ones. ${ }^{9}$ There was a 3.2 -fold higher risk of preterm birth and a 2-fold higher risk of preterm birth in IVF pregnancies compared to spontaneous singleton pregnancies. In terms of newborn weight, the results show a 3-fold higher risk of very low birth weight and a 1.7-fold higher risk of low birth weight, as well as a 1.4-fold higher risk for SGA. The risk of NICU admission is 1.27 times higher in children born after IVF pregnancy than in those after spontaneous 
conception. These results demonstrate that assisted reproduction is a predictor of preterm birth that should be considered when monitoring these pregnancies. This effect is more pronounced in early preterm births and leads to a higher percentage of children born with low and very low birth weight, NICU admission and higher perinatal mortality. Also, $40 \%$ more SGA newborns are observed after IVF. ${ }^{9}$

As the problems of ART pregnancies continue to be relevant today, their study continues. A very large analysis examines all available studies on IVF pregnancies and their outcome by August 2016. Emphasis is placed on the fact that any stage of high-tech infertility treatment can affect the risks of pregnancy and perinatal complications. ${ }^{10}$ On the other hand, a large study showed that the outcome of birth was not significantly different, except that the birth occured on average 3 days earlier in pregnancies after IVF compared to spontaneous pregnancies. Birth weight is slightly lower in the IVF group, but the neonatal outcome in term infants is similar. ${ }^{11}$ One study in Israel showed that the incidence of PB, LBW, SGA, NICU admission and perinatal mortality were comparable in singleton pregnancies after IVF and those that occurred spontaneously. ${ }^{12}$

\section{Number of embryos transferred during IVF procedure}

Singleton pregnancy with a live birth beyond 37 weeks of gestation is the ultimate goal of any assisted reproductive technology. It was hypothesized that the outcome of singleton conceived after transfer of two or more embryos may be dependent on the sharing of uterine space with other embryos. There is higher chance of missed abortion in patients with singleton pregnancies conceived after multiple embryo transfer. Gestational age at delivery and birth weight are correlated with number of embryos transfered. ${ }^{13} \mathrm{~A}$ large meta-analysis examined whether the return of one embryo could reduce the higher risk of poor perinatal outcome associated with IVF compared on the one hand with the transfer of two or more embryos and on the other with spontaneous conception. It has been shown that the risk of preterm birth and low birth weight is lower in singleton pregnancies achieved by transferring one embryo than in those achieved by transferring two or more embryos. At the same time, compared to spontaneous singleton pregnancies, those after IVF with embryo transfer of one embryo carry a higher risk of preterm birth. ${ }^{14}$ Some authors believe that the outcome of pregnancies in IVF singleton pregnancies with transfer of one embryo does not differ significantly from that in spontaneous singleton pregnancies. ${ }^{15}$

\section{Presence of a "vanishing twin"}

In approximately $10 \%$ of singleton pregnancies achieved after double embryo transfer, the development of one embryo stops (the socalled "vanishing twin") and these pregnancies continue as singleton, with one child being born. ${ }^{16}$ These pregnancies have an increased risk of preterm birth and low birth weight compared to singleton from the beginning IVF pregnancies. ${ }^{17-19}$ Some authors found that vanishing twin pregnancies had a lower prevalence and a worse perinatal outcome after IVF-ICSI as compared with those of their spontaneously conceived counterparts. ${ }^{20}$ It is possible that a poorer neonatal outcome in the presence of a vanishing twin contributes to a poorer overall outcome in singleton IVF pregnancies.

\section{Type of embryo transfer}

The number of children born after embryo transfer of frozen / thawed embryos is constantly increasing. Singleton pregnancies achieved through the transfer of frozen embryos are considered to carry a lower risk of preterm birth, low birth weight and SGA compared to those after stimulated IVF and 'fresh' embryo transfer. ${ }^{21}$ Some even find a similar outcome of FET pregnancies compared to spontaneous singleton pregnancies in terms of the incidence of PB, LBW and SGA, while others find a higher incidence of these indicators in IVF-FET pregnant women compared to spontaneous pregnancies. ${ }^{22}$ It is suggested that more physiological preparation of the endometrium before FET is related to a better pregnancy outcome, possibly providing better placentation and early embryonic development than that of stimulated cycles.

Most likely, both IVF and infertility are responsible for the worse outcome of the pregnancy. Which of the two factors has a dominant influence is difficult to fully-understand. When analyzing the results of "low-tech" treatment of infertility (intrauterine insemination, donor insemination), IVF and spontaneous conception, it is found that preterm birth is observed $50 \%$ more often in the first group and twice as often after IVF, proving the role of two factors - infertility itself and IVF - for the higher incidence of preterm birth. ${ }^{23}$ Others found no differences in pregnancy outcome after IUI and IVF. Therefore it can be concluded that the poorer outcome of IVF pregnancy is due to maternal factors and not to the IVF procedure itself. ${ }^{24,25} \mathrm{~A}$ small study found increased incidence of PTB, LBW and SGA in IVF pregnancies when compared with IUI singleton pregnancies. ${ }^{26}$

\section{Complications of pregnancy \\ Preeclampsia and gestational diabetes}

The use of IVF is associated with a higher incidence of some complications of pregnancy, some of which include the development of preeclampsia ${ }^{27-29}$ and gestational diabetes. ${ }^{27,30-32}$ A large retrospective cohort study investigated the correlation between IVF and preeclampsia and their combined impact on pregnancy outcomes. The risk of developing PE in pregnancies following IVF was more than two-fold higher compared to spontaneous conception. In preeclamptic patients without a definite risk factor for PE, singleton mothers with IVF were more likely to suffer from severe disease and concomitant complications such as GDM, hypothyroidism and placenta accrete compared to singleton mothers with spontaneous conception. ${ }^{27}$ There are large analyzes proving conclusively the link between IVF and the development of both preeclampsia and gestational diabetes in multiple and singleton pregnancies. When comparing singleton pregnancies after IVF with spontaneous singleton pregnancies there can be concluded that the risk of developing hypertensive complications is 1.49 times higher in pregnancies after IVF. There is a 1.48 times higher risk of developing gestational diabetes in IVF pregnancies compared to the pregnancies with spontaneous conception. When analyzing the course of pregnancies achieved through IVF, the question often arises as to whether the complications are due to the IVF procedure or to the characteristics of the infertile couples undergoing the specific treatment. After considering factors such as age, parity, and limitation to singleton pregnancies alone, some studies have shown an increased risk of developing preeclampsia in women after infertility treatment, ${ }^{28,33,34}$ while others have found no such dependence. ${ }^{35-37}$

\section{Placenta previa}

Obstetric blood loss and primary postpartum blood loss are the leading cause of maternal mortality worldwide. The leading causes are complications of pregnancy associated with placental pathology - placenta previa and placental abruption. The etiology of placenta previa is not always clear. There are a number of known factors leading to the development of this disease. Most often these are age 
over 35 years, multiparity, multiple pregnancy, short interval between pregnancies, previous uterine surgery, previous cesarean section, previous or recurrent abortions, treatment of infertility, placenta previa in previous pregnancy, non-white race, low socioeconomic status, smoking, cocaine use. ${ }^{38-40}$

An interesting question is the frequency of placenta previa in pregnancies achieved by assisted reproductive technologies. There are a number of large studies that unequivocally prove that placenta previa is a complication that is more common among pregnancies achieved through IVF. ${ }^{38-43}$ A meta-analysis of six small studies found a threefold higher risk of placenta previa after IVF. ${ }^{8}$ Most authors try to answer the question which exact element of the In Vitro procedure brings the most-highest risk - ovarian hyperstimulation, laboratory part, embryo transfer, number of embryos transferred, type of embryo transfer, etc. Risk factors from the mother's condition such as age, parity, bad habits, previous cesarean sections, etc. are also analyzed. Some authors found a six-fold higher incidence of placenta previa in IVF singleton pregnancies than controls in spontaneous singleton pregnancies. ${ }^{28}$ There are some suggestions that blastocyst transfer may be more likely to lead to placenta previa than transfer to an earlier stage of division. ${ }^{41}$ The incidence of both placenta previa and associated antepartum hemorrhage may be reduced in pregnancies resulting from the transfer of frozen/thawed embryos instead of fresh embryos, which may be due to the fact that in these cases the endometrium is not subject to strong hormonal stimulation as in stimulated cycles. ${ }^{39}$

\section{IVF and delivery}

Birth planning for women who become pregnant after IVF is an important part of caring for couples with reproductive problems. The decision on the timing and manner of delivery should take into account the various risk factors preceding pregnancy, as well as the presence of pregnancy complications. The incidence of elective caesarean sections at the patient's request is increased in most countries in pregnancies after IVF, with percentages exceeding $50 \%$ in some countries. This is particularly common in older patients, where the fear of a poor fetal outcome lowers the decision threshold for interventions such as induction of labor and operative delivery. ${ }^{44-49}$

Most studies show that the frequency of cesarean sections after IVF is increased compared to the frequency after spontaneous conception. This applies to both multiple and singleton pregnancies. ${ }^{28,30,32,44,45-49}$ The higher frequency of cesarean sections in IVF pregnancies requires that the decision on the method of delivery after IVF must be motivated. In Australia, between 2003 and 2005, the incidence of cesarean sections among IVF pregnancies was $50.1 \%$, compared with $28.9 \%$ among the general population. In singleton pregnancies after IVF, the incidence of cesarean section was $43.8 \%$ versus $27.8 \%$ in the general population. The incidence of cesarean section appears to be disproportionately higher in singleton term pregnancies. This requires that vaginal birth must be supported and that indications for cesarean section must be medically motivated. ${ }^{47}$ Some authors found increased rate of breech presentation in IVF singleton pregnancies that can influence the cesarean section rate. ${ }^{50,51}$ On the other hand, some have found that even in cases with a favorable pelvic score and despite parity, the cesarean section rate was much higher in IVF pregnancies compared to spontaneous ones. ${ }^{52}$

A number of studies have found a worse outcome for the newborn after IVF. $89,32,53-56$ The reasons may be due to the higher frequency of preterm birth in IVF pregnant women, as well as some complications of pregnancy. ${ }^{57}$ The stay of the newborn in NICU is more frequent ${ }^{8,9,51}$ and the perinatal mortality is increased. ${ }^{9,30,32,54,55,58}$ Some researchers found, that most of the risks for the IVF newborns are mainly related to increased rate of multiple pregnancies. ${ }^{59}$ More often in IVF singleton deliveries are observed SGA newborns. ${ }^{6,8,11,30,55,60}$ The development of SGA in IVF in pregnant women is influenced by factors such as controlled ovarian hyperstimulation, the transfer of frozen embryos and the presence of a "vanishing twin", which were discussed earlier. Some authors found a link between SGA and the presence of a vanishing twin in singleton pregnancies after IVF. ${ }^{61}$ SGA has been shown to be a more common problem in singleton pregnancies with a vanishing twin compared to singleton pregnancies from the beginning, and this difference increases the later the cessation of the development of the second fetal sac occurs. The difference persists even after considering a number of risk factors for SGA hypertension in pregnancy, preeclampsia, HELLP syndrome, placenta previa, placental abruption, bleeding in the first and third trimesters of pregnancy. ${ }^{62}$

\section{Conclusion}

Singleton pregnancies after In vitro fertilization are distinguished by a number of peculiarities in their course. The average age of pregnant women after IVF is higher than the age in the general population. They are more likely to have preterm birth, low birth weight and small for gestational age neonates. Factors that worsen the prognosis are the higher number of transferred embryos, the presence of a vanishing twin and controlled ovarian stimulation. Some pregnancy complications such as preeclampsia, gestational diabetes and placenta previa are also more common in IVF pregnancies. Pregnant women are more likely to give birth by caesarean section and the proportion of newborns who need intensive care is higher. Future studies are needed to improve the prognosis in these pregnancies.

\section{Acknowledgments}

None.

\section{Funding}

None.

\section{Conflicts of interest}

None.

\section{References}

1. Allen VM, Halifax NS, Wilson RD, et al. Pregnancy Outcomes After Assisted Reproductive Technology. Joint SOGC-CFAS Guideline. 2006;28(3):220-223.

2. Slavov S. Obstetric problems in second half of pregnancy and delivery in singleton pregnancies achieved by In vitro fertilization[dissertation]. Sofia, Bulgaria: Medical University. 2020; 62.

3. Jacobsson B,Ladfors L, Milsom I. Advanced maternal age and adverse perinatal outcome. Obstet Gynecol. 2004;104(4):727-733.

4. Ban Frangez H, S Korosec, I Verdenik, et al. Preterm delivery risk factors in singletons born after in vitro fertilization procedures. Eur J Obstet Gynecol Reprod Biol. 2014;176:183-186.

5. Slavov S, Zlatkov G, Nikolov A. Preterm labor in singleton pregnancies achieved by In vitro fertilization. Akusherstvo i ginekologia. 2019;58(1):915 .

6. Slavov S, Neykova K, Ingilizova G, et al. The incidence of small for gestational age neonates in singleton IVF pregnancies. The Journal of Maternal-Fetal \& Neonatal medicine. 2021;1-3. 
7. Chung K, Coutifaris C, Chalian R, et al. Factors influencing adverse perinatal outcomes in pregnancies achieved through use of in vitro fertilization. Fertility and Sterility. 2006;86(6):1634-1641.

8. Jackson RA, Gibson KA, Wu YW, et al. Perinatal outcomes in singletons following in vitro fertilization: a meta-analysis. Obstet Gynecol. 2004;103(3):551-563.

9. Helmerhorst FM, Perquin DA, Donker D, et al. Perinatal outcome of singletons and twins after assisted conception:a systematic review of controlled studies. BMJ. 2004;328(7434):261.

10. Polomba S, Homburg R, Santagni S, et al. Risk of adverse pregnancy and perinatal outcomes after high technology infertility treatment: a comprehensive systematic review. Reproductive Biology and Endocrinology. 2016;14(1):76.

11. Koudstaal J, Braat DDM, Bruinse HW, et al. Obstetric outcome of singleton pregnancies after IVF: a matched control study in four Dutch university hospitals. Human Reproduction. 2000;15(8):1819-1825.

12. Reubinoff BE, Samueloff A, Ben-Haim M et al. Is the obstetric outcome of in vitro fertilized singleton gestations different from natural ones? A controlled study. Fertility and Sterility. 1997;67(6):1077-1083.

13. Bhandari S, Ganguly I, Agarwal P, et al. Relationship of Number of Embryos Transferred with Perinatal Outcome of Singleton Pregnancy. $J$ Reprod Infertil. 2017;18(1):179-184.

14. Grady R, Alavi N, Vale R et al. Elective single embryo transfer and preinatal outcomes: a systematic review and meta-analyses. FertilSteril, 2012;7(2):324-331.

15. Neubourg D, Mangelschots K, Van Royen E, et al. The obstetrical and neonatal outcome of babies born after single-embryo transfer in IVF/ ICSI compares favourably to spontaneously conceived babies. Human Reproduction. 2006;21(4):1041-1046.

16. Pinborg A, Lidegaard O, Freiesleben N, et al. Consequences of vanishing twins in IVF/ICSI pregnancies. Human Reproduction. 2005;20(10):28212829

17. Dickey RP, Taylor SN, Lu PY, et al. Spontaneous reduction of multiple pregnancy: Incidence and effect on outcome. Am J Obstet Gynecol. 2002;186(1):77-83

18. Schieve L, Ferre C, Peterson H, et al. Perinatal outcome among singleton infants conceived through assisted reproductive technology in the United States. Obstetrics \& Gynecology. 2004;103(6):1144-1153.

19. Slavov S, Karamisheva V, Nikolov A. Influence of vanishing twin syndrome on outcome of singleton pregnancies achieved by In vitro fertilization. Comptesrendus de l'Academie bulgare des Sciences. 2021;74(6):914-919.

20. Márton V, Zádori J, Kozinszky Z, et al. Prevalences and pregnancy outcome of vanishing twin pregnancies achieved by in vitro fertilization versus natural conception. Fertil Steril. 2016;106(6):1399-1406.

21. Maheshwari A, Raja EA, Bhattacharya S. Obstetric and perinatal outcomes after either fresh or thawed frozen embryo transfer: an analysis of 112,432 singleton pregnancies recorded in the Human Fertilisation and Embryology Authority anonymized dataset. FertilSteril. 2016;106(7):1703-1708.

22. Wennerholm UB, Henningsen AK, Romundstad LB, et al. Perinatal outcomes of children born after frozen-thawed embryo transfer: a Nordic cohort study from the CoNARTaS group. Human Reproduction. 2013;28(9):2545-2553.

23. Wang JX, Norman RJ, Kristiansson P. The effect of various infertility treatments on the risk of preterm birth. Human Reproduction. 2002;17(4):945-949.

24. De Sutter P, Valdeman L, Kok P, et al. Comparison of outcome of pregnancy after intra-uterine insemination (IUI) and IVF. Human Reproduction. 2005;20(6):1642-1646.
25. Messerlian C, Platt RW, Tan SL, et al. Low-technology assisted reproduction and the risk of preterm birth in a hospital-based cohort. Fertility and Sterility. 2014;103(1):81-88.

26. Slavov S. Impact of different methods of conception on the outcome of pregnancy in infertile women. Akusherstvo i ginekologia.

27. Gui J, Ling Z, Hou X, et al. In vitro fertilization is associated with the onset and progression of preeclampsia. Placenta. 2020;89:50-57.

28. Shevell T, Malone F, Vidaver J, et al. Assisted reproductive technology and pregnancy outcome. Obstetrics\&Gynecology. 2005;106(5):10391045 .

29. Slavov S, Nikolov A. Obstetric complications in singleton pregnancies achieved by In vitro fertilization. Akusherstvo i ginekologia. 2020;59(1):37.

30. Pandey S., Shetty A, Hamilton M et al. Obstetric and perinatal outcomes in singleton pregnancies resulting from IVF/ICSI: a systematic review and meta-analysis. Human Reproduction Update. 2012;18(5):485-503.

31. QinJ, LiuX, Sheng Hetal. Assisted reproductive technology and the risk of pregnancy-related complications and adverse pregnancy outcomes in singleton pregnancies: ameta-analysis of cohort studies. Fertil Steril. 2016;105(1):73-85.

32. Silberstein T, Levy A, Herlev A, et al. Perinatal outcome of pregnancies following in vitro fertilization and ovulation induction. J Matern Fetal Neonatal Med. 2014;27(13):1316-1319.

33. Tabs D, Vejnocic T, Radunovi N. Pre-eclampsia and eclampsia in parturients from the in vitro fertilization program. Med Pregl. 2004;57(12):7-12.

34. Tanbo T, Dale PO, Lunde O, et al. Obstetric outcome in singleton pregnancies after assisted reproduction. Obstet Gynecol. 1995;86(2):188192.

35. Sun M, Walker MC, Cao HL et al. Assisted reproductive technology and placenta-mediated adverse pregnancy outcomes. Obstetrics\& Gynecology. 2009;114(4):818-824.

36. Caserta D., Marci R., Tatone C. et al. IVF pregnancies: neonatal outcomes after the new Italian law on assisted reproduction technology (law 40/2004). Acta Obstet Gynecol Scand. 2008;87(9):935-939.

37. Olivennes F, Rufat P, Andre A et al. Pregnancy: The increased risk of complication observed in singleton pregnancies resulting from in-vitro fertilization (IVF) does not seem to be related to the IVF method itself. Hum Reprod. 1993;8(8):1297-1300.

38. Hayashi M, Nakai A, Satoh S, et al. Adverse obstetric and perinatal outcomes of singleton pregnancies may be related to maternal factors associated with infertility rather than the type of assisted reproductive technology procedure used. Fertil Steril, 2012;98(4):922-928.

39. Korosec S, Frangez HB, Verdenik I, et al. Singleton pregnancy outcomes after in vitro fertilization with fresh or frozen-thawed embryo transfer and incidence of placenta praevia. BioMed Research international. 2014:431797

40. Raisanen S, Rendell K, Nielsen HS, et al. Socioeconomic status affecting the prevalence, but not the perinatal outcomes, of in vitro fertilization pregnancies. Hum Reprod. 2013;28(11):3118-3125.

41. Sazonova A, Kallen K, Thurin-Kjellberg A, et al. Obstetric outcome in singletons after in vitro fertilization with cryopreserved/thawed embryos. Hum Reprod. 2012;27(5):1343-1350.

42. Slavov S, Zlatkov G, Nikolov A. Pathology of the placenta in singleton pregnancies achieved by In vitro fertilization. Akusherstvo i ginekologia, 2019;58(1):3-9.

43. Yang X, Li Y, Zhang W. Current overview of pregnancy complications and live-birth outcome of assisted reproductive technology in mainland China. Fertil Steril. 2014;101(2):385-391. 
44. Verlaenen H, Cammu H, Derde MP. Singleton pregnancy after in-vitro fertilization: expectations and outcome. Obstetrics and Gynecology. 1995;86(6):906-910.

45. Vilchez G, Dai J, Hoyos LR, et al. Optimal delivery mode in singleton pregnancies conceived after infertility treatment: is the "precious baby" effect justified? American journal of Obstetrics \& Gynecology. 2016;suppl.:445-446.

46. Apantaku O, Chandrasekaran I, Bentick B. Obstetric outcome of singleton pregnancies achieved with in vitro fertilization and intracytoplasmic sperm injection: Experience from a district general hospital. Journal of Obstetrics and Gynaecology. 2008;28(4):398-402.

47. Sullivan E, Chapman M, Wang YA et al. Population-based study of cesarean section after in vitro fertilization in Australia. BIRTH. 2010;37(3):184-191

48. Kallen B, Finnstrom O, Nygren KG, et al. In vitro fertilization in Sweden: obstetric characteristics, maternal morbidity and mortality. BJOG. 2005;112(11):1529-1535

49. Perri $\mathrm{T}$, Chen $\mathrm{R}$, Yoeli $\mathrm{R}$ et al. Are singleton assisted reproductive technology pregnancies at risk of prematurity? Journal of assisted reproduction and genetics. 2001;18(5):245-249.

50. Slavov S. Obstetric problems in second half of pregnancy and delivery in singleton pregnancies achieved by In vitro fertilization[dissertation] Sofia, Bulgaria: Medical University; 2020;118.

51. Romundstad LB, Romundstad PR, SundeA et al. Assisted fertilization and breech delivery: risks and obstetric management. Human Reproduction. 2009;24(12):3205-3210.

52. Slavov S, Nikolov A. Cesarean section in singleton pregnancies achieved by In vitro fertilization. Akusherstvo i ginekologia, 2019; 58(2):18-22.

53. Klemetti R, Gissler M. Comparison of perinatal health of children born from IVF in Finland in the early and late 1990s. Human Reproduction. 2002;117(8):2192-2198.
54. Draper E, Kurinczuk JJ, Abrams KR, et al. Assessment of separate contributions to perinatal mortality of infertility history and treatment: a case-control analysis. The Lancet. 1999;353(9166):1746-149.

55. Marino J, Moore VM, Willson K, et al. Perinatal Outcomes by Mode of Assisted Conception and Sub-Fertility in an Australian Data Linkage Cohort. PLOS One. 2014;9(1):e80398.

56. Wisborg K, Ingerslev HJ, Henriksen TB. In vitro fertilization and preterm delivery, low birth weight, and admission to the neonatal intensive care unit: a prospective follow-up study. Fertility and Sterility. 2010;94(6):2102-2106

57. Slavov S, Zlatkov G, Nikolov A. Neonatal outcome in singleton pregnancies achieved by in vitro fertilization. Akusherstvo $i$ ginekologia, 2021;60(1):7-11.

58. Kostov I, Ingilizova G. Analysis of the results of inspections carried out by executive agency "Medical Audit" of Neonatology practice in Bulgaria. Obstetrics and Gynaecology, 2019;58(1):16-20.

59. Vakrilova L, Slavov S, Hitrova S, et al. Problems and neonatal outcome of very low birth weight newborn infants after in vitro fertilization. Akusherstvo i ginekologia. 2013;52(1):30-34.

60. McDonald S., Han Z, Mulla S, et al. Preterm birth and low birth weight among in vitro fertilization singletons: A systematic review and metaanalyses. European Journal of Obstetrics \& Gynecology and Reproductive Biology. 2009;146(2):138-148.

61. Pinborg A, Lidegaard O, Freiesleben N, et al. Vanishing twins: a predictor of small-for-gestational age in IVF singletons. Human Reproduction. 2007;22(10):2707-2714.

62. Kostov I. Assessment of the Impact of Medical Standard in Obstetrics and Gynecology: Organizational, Clinical and Deontological Aspects. Doctoral Thesis. Varna, 2017. 